J. Clin. Chem. Clin. Biochem.

Vol. 18, 1980, pp. 879-883

\title{
An Evaluation Method Providing Confidence Intervals Applied to Radioimmunoassay
}

\author{
By L. Lindwall
}

Dept. of Mathematics, Linköping University, Linköping,

\section{Molin}

Dept. of Clinical Pharmacology and

\section{B. Bergdahl}

Dept. of Internal Medicine, University Hospital, Linköping, Sweden

(Received October 1, 1979/May 12, 1980)

Summary: A method for evaluation of radioimmunoassay results is described. The order of the single tubes in each assay run is randomized. A polynomial is fitted to untransformed data ( $y=$ counts per minute; $x=$ concentration of the compound assayed) by regression. The concentrations of unknown samples are evaluated by interpolation to the curve. A confidence interval is calculated for each sample, taking into account the variance of the standard curve and that of the actual duplicate assay jointly.

\section{Anwendung einer Auswertungsmethode zur Gewinnung von Vertrauensbereichen auf Radioimmunassays}

Zusammenfassung: Eine Methode zur Auswertung von Ergebnissen von Radioimmunassays wird beschrieben. Die Reihenfolge der einzelnen Probengefäße in jeder Bestimmungsserie wird dem Zufall überlassen. Ein Polynom wird durch Regression auf nicht-transformierte Daten ( $y=$ Zählrate; $x=$ Konzentration der gemessenen Substanz) angepaßt. Die Konzentrationen der unbekannten Proben werden durch Interpolation zur Kurve ermittelt. Ein Vertrauensbereich für jede Probe wird unter gemeinsamer Berücksichtigung der Varianz der Standardkurve sowie der der aktuellen Doppelbestimmung berechnet.

\section{Introduction}

Automated calculation of radioimmunoassay (RIA) results seems preferable to manual (1). The two major approachés used for curve-fitting in RIA are modelbased $(2,3,4)$ and data-based $(5,6,7)$; a good review of functions used was given by Vogt et al. (8). Factors in RIA, such as continuing saturation of the assayed compound, inhomogeneity of antibody populations, cross reactions ānd nonspecific protein interference $(6,9,10)$ generally invalidate simple models based on the law of mass action. Complicated models based on the law of mass action are of interest for studying the theory of RIA, but due to computational complexity they are less attractive for routine use. Linearizing methods based on the law of mass action can be evaluated by least square linear regression, and especially the logit-log method (2) is frequently used due to its simplicity. However, this method involves a risk of errors, if a correct weighting of data is not made (11) and has been subject to criticism $(12,13,14)$. Therefore data-based universal methods where spline functions are used $(6,14,15,16)$ seem preferable.

Since the precision of RIA is rather low (17) we and others $(1,18)$ think that an evaluation method should also give an estimate of the reliability of the result. In the case of spline functions, such calculations are most complex. Our aim was to design a simple data-based method for RIA-evaluation providing confidence intervals. For curve-fitting we used a polynomial to fit untransformed data. Confidence limits were calculated using a version of the Fieller-theorem (19).

\section{Method}

We assume that the relationship between concentration and the registered counts per minute (cpm) can be 
described as a function of the concentration (c) plus a random error $(\epsilon)$. Thus:

$$
\mathrm{cpm}=\mathrm{f}(\mathrm{c})+\epsilon
$$

The random error is assumed to be normally distributed with zero mean and variance $\sigma_{\epsilon}^{2}$ and uncorrelated with c. $f(c)$ is approximated with the $p^{\text {th }}$ degree polynomial in $\mathrm{c}$ :

$$
f(c)=\beta_{0}+\beta_{1} c+\beta_{2} c^{2}+\ldots+\beta_{p} c^{p}
$$

The degree of the polynomial can be chosen from a visual inspection of a plot of the actual data. In the case of logit-log transformed data, a first degree polynomial, i.e. a straight line, can be used.

An assay run consists of $\mathrm{M}$ standard samples tubes with known, and $\mathrm{N}$ tubes with sample of unknown concentrations. The $\mathrm{M}$ and $\mathrm{N}$ tubes are placed randomly in the run. The number of unknown concentrations in one assay run is denoted $q$, and the number of single tubes with unknown concentration $v_{i}$ is denoted $n_{\mathbf{i}}$. Thus:

$$
\sum_{i=1}^{q} n_{i}=N
$$

$X$ represents the $M(p+1)$ matrix of known concentrations and their powers:

$$
X=\left[\begin{array}{ccccc}
1 & c_{1} & c_{1}^{2} & \ldots & c_{1}^{p} \\
1 & c_{2} & c_{2}^{2} & \ldots & c_{2}^{p} \\
\vdots & & & \vdots \\
1 & c_{M} & c_{M}^{2} & & c_{M}^{p}
\end{array}\right]
$$

$\mathrm{X}^{*}$ denotes the transpose of $\mathrm{X}$.

$$
\mathrm{X}^{*}=\left[\begin{array}{cccc}
1 & 1 & \ldots & 1 \\
c_{1} & c_{2} & \ldots & c_{M} \\
c_{1}^{2} & c_{2}^{2} & \ldots & c_{M}^{2} \\
\vdots & & \vdots \\
c_{1}^{p} & c_{2}^{p} & \ldots & c_{M}^{p}
\end{array}\right]
$$

$\mathrm{Y}$ represents the column vector of observed cpm-values corresponding to the $M$ known concentrations.

$$
\mathrm{Y}=\left[\begin{array}{c}
\mathrm{y}_{1} \\
\mathrm{y}_{2} \\
\vdots \\
\mathrm{y}_{\mathrm{M}}
\end{array}\right]
$$

Let

$$
C=\left(X^{*} X\right)^{-1}
$$

i.e. the inverse of the matrix $\left(X^{*} X\right)$. We can then easily obtain a least square estimate of the betas $(\beta: s)$ in
Eq. (2) and of $\sigma_{\epsilon}^{2}$ by ordinary multiple regression. The column vector $B$ of estimated $\beta: s$ is then given by the expression:

$$
\mathrm{B}=\mathrm{C} \mathrm{X}^{*} \mathrm{Y}
$$

The estimate $\mathrm{s}_{\epsilon}^{2}$ of $\sigma_{\epsilon}^{2}$ is given by:

$$
s_{\epsilon}^{2}=\frac{\sum_{i=1}^{M}\left(b_{0}+b_{1} c_{i}+b_{2} c_{i}^{2}+\ldots+b_{p} c_{i}^{p}-y_{i}\right)^{2}}{M-p-1}
$$

where $y_{i}, i=1,2, \ldots, M$ are the elements of $Y$ and $b_{j}$, $j=0,1,2, \ldots, p$ the elements of $B$. This variance estimate has $M-p-1$ degrees of freedom. Obviously, the number of different concentrations ( $r$ ), in which the $M$ calibration points are placed must be chosen so that

$$
r \geqslant p+1
$$

If the polynomial Eq. (2) does not exactly represent the true relationship between $\mathrm{cpm}$ and $\mathrm{c}$, the estimate $\mathrm{s}_{\epsilon}^{2}$ will be biased. In this case, the tendency will usually be to overestimate $\sigma_{\epsilon}^{2}$ slightly. As an alternative, we may use an estimate of $\sigma_{\epsilon}^{2}$ with more degrees of freedom than Eq. (8) namely:

$s_{\epsilon}^{2}=\frac{\sum_{i=1}^{M}\left(b_{0}+b_{1} c_{i}+b_{2} c_{i}^{2}+\ldots+b_{p} c_{i}^{p}-y_{i}\right)^{2}+\sum_{i=1}^{q} \sum_{\ell=1}^{n_{i}}\left(a_{i \ell}-\overline{\bar{a}}_{i}\right)^{2}}{M-p-1+N-q}$

where $a_{i \ell}$ is the observed cpm of the 1 st tube with concentration $v_{i}$ and $\bar{a}_{i}$ is the mean value of the observed $\mathrm{cpm}: \mathrm{s}$ of the concentration $v_{\mathbf{i}}$. This variance estimate has $M-p-1+N-q$ degrees of freedom.

The problem of dealing jointly with the random error of the standard curve and the random error of the single sample in order to obtain a confidence interval for the unknown concentration $v_{i}$ can be solved by regarding the quantity:

$$
\bar{a}_{i}-\left(b_{0}+b_{1} v_{i}+b_{2} v_{i}^{2}+\ldots+b_{p} v_{i}^{p}\right)
$$

The expectation of Eq. (11) is apparently $=0$ and since $\overline{\mathrm{a}}_{\mathrm{i}}$ is not correlated to the other terms of the equation the variance of Eq. (11) can be written:

$$
\begin{aligned}
& \sigma^{2}\left(\bar{a}_{i}\right)+\sigma^{2}\left(b_{0}+b_{1} v_{i}+b_{2} v_{i}^{2}+\ldots b_{p} v_{i}^{p}\right)= \\
& =\frac{\sigma_{\epsilon}^{2}}{n_{i}}+\sigma^{2}\left(b_{0}+b_{1} v_{i}+b_{2} v_{i}^{2}+\ldots+b_{p} v_{i}^{p}\right)
\end{aligned}
$$


In this expression the first term can be estimated with $s_{\epsilon}^{2} / n_{i}$ and the second with $s_{\epsilon}^{2} V_{i}^{*} C V_{i}$, where $V_{i}$ is the column vector

$$
V_{i}=\left[\begin{array}{c}
1 \\
v_{i} \\
v_{i}^{2} \\
\vdots \\
v_{i}^{p}
\end{array}\right]
$$

$V_{i}^{*}$ is the transpose of $V_{i}$ and $C$ is defined by Eq. (6).

As a consequence the inequality:

$$
-t_{\alpha / 2} \leqslant \frac{\bar{a}_{i}-\left(b_{0}+b_{1} v_{i}+b_{2} v_{i}^{2}+\ldots+b_{p} v_{i}^{p}\right)}{\sqrt{s_{\epsilon}^{2}\left(\frac{1}{n_{i}}+v_{i}^{*} C v_{i}\right)}} \leqslant t_{\alpha / 2}
$$

where $t_{\alpha / 2}$ is the $\alpha / 2$ quantile in the Student's t-distribution, and holds for $\alpha 100 \%$ of all samples. The number of degrees of freedom is achieved from Eq. (8) or Eq. (10) depending on the estimate of $\sigma_{\epsilon}^{2}$ utilized in Eq. (13). Since $v_{i}$ is the only unknown quantity in Eq. (13) the set of $v_{i}: s$ for which the inequality holds forms a $\alpha$ $100 \%$ confidence interval for the unknown concentration $v_{i}$. The confidence limits may be sought using more or less sophisticated methods. One way is simply to try different values of $v_{i}$ and look for values of it that lead to either left hand or right hand equality in Eq. (13).

There are several ways of obtaining a point estimate of $\mathbf{v}_{\mathbf{i}}$. For example, the midpoint of the confidence interval described above or the $v_{i}$ that makes the numerator of Eq. (13) equal to zero.

\section{Applications and Discussion}

The present evaluation method has been applied to commercial RIAs for digoxin representing different assay systems. Six or seven standard levels were used in duplicate. Since the lower limit of detection of digoxin RIAs is usually $0.3-0.7 \mathrm{nmol} / 1(20)$, the zero calibration level might be excluded to obtain a better fit of the function. A second degree polynomial mostly gave multiple regression coefficients ( $r$ ) of $0.98-0.99$; r-values less than 0.98 were not accepted. Lower r-values were due to large variation in the measured bound counts of the standards and not to a bad fit of the polynomial. For example, in one case we identified a clear outlier as being the reason for an $r$-value of 0.91 . When this value was left out, $r$ was 0.996 . Two standard curves and examples of $95 \%$ confidence intervals for unknown concentrations are given in figure 1.

Errors in RIA are often incompletely controlled and may vary from assay to assay. To eliminate bias from systematic errors, we used a randomized order of all single tubes (standards and unknowns together) in an assay series. There were no major difficulties or waste of time in the implementation of such a design.

A simple polynomial to approximate the standard curve might be regarded as an oversimplification. Other functions, especially the logit-log type have been stated to be superior to polynomials $(5,21)$. However, an empirical function that is not quite appropriate seems acceptable in RIA, since the random errors involved are apparently much greater than those introduced by the use of such a function (22). This suggestion is also supported by our
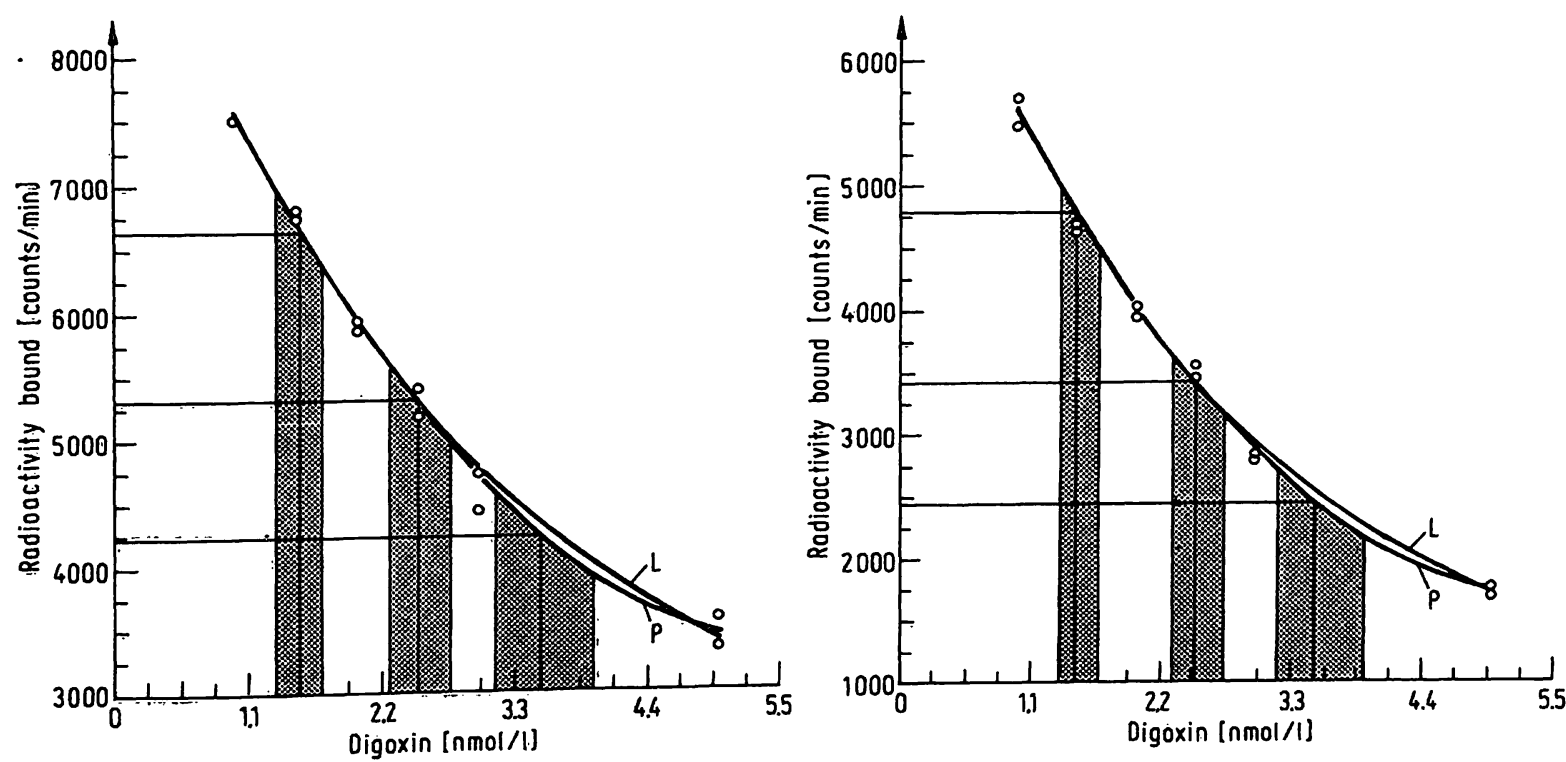

Fig. 1. Plots of $\mathrm{cpm}$ for bound fraction versus concentration (nmol/l) from one assay run each with Gammacoat ${ }^{125}$ I Digoxin RIA kit from centrations $(1.5,2.5$ and $3.5 \mathrm{nmol} / \mathrm{l})$ are indicated. $O=$ calibration points, $P=$ fitted $2 \mathrm{nd}$ degree polynomial and $\mathrm{L}=$ fitted logistic function. 
results. As illustrated in figure 1 , the difference between the fitted second degree polynomial and an unweighted logistic function corresponding to the logit-log method recommended by others $(3,23)$ is small compared to the width of the $95 \%$ confidence intervals. Moreover, in another study (24) the results obtained with the present method for evaluating digoxin RIAs did not differ appreciably from those obtained manually according to the kit instructions.

The assumption that sampling errors are normally distributed and not correlated to concentration can also be questioned. The emission of radioactivity follows a Poisson distribution rather than a normal one and its variance depends on its intensity. However, this Poisson distribution is associated with a very high intensity, and can thus be quite well approximated with, a normal distribution (25). It can also be seen in figure 1 that the variation in absolute counts was quite small along the concentration axis and there is solid documentation that least squares methods are robust against slight deviations from their assumptions (26). Moreover, if the deviation from our assumption regarding variance were not quite negligible, the main implication would only be an underestimation of the width of the confidence intervals at low and an overestimation at high concentrations. In our opinion, there is no reason to believe that other known components of the total error in RIA, as reviewed by e.g.Ekins (27), are correlated to concentration.

To study the influence of nonconformity in variance on the validity of the results, the outcome of the RIAs presented in figure 1 were simulated in a computer. The random error was assumed to consist of one Poisson component with variance equal to its mean and one normally distributed component with the same variance as the former at the lowest standard concentration. Calibration points for 1000 standard curves were generated using a second degree polynomial $(\mathrm{P})$ and a logistic function (L) according Pilo \& Zucchelli (23). For each curve, cpm values for the three samples assayed in dupli- cate and with true concentrations of 1.5, 2.5 and $3.5 \mathrm{nmol} / \mathrm{l}$, respectively, were simulated. For $P$ the number of confidence intervals containing the true concentrations were $94.9 \%, 95.2 \%$ and $94.9 \%$, respectively. The corresponding figures for $\mathrm{L}$ were $95.4 \%, 94.4 \%$ and $97.7 \%$, respectively. Thus, for both functions the stipulated $95 \%$ confidence was very closely obtained.

The Fieller-theorem is considered the best statistical approach for calculation of confidence intervals, which also include the component due to curve-fitting $(4,28)$. In view of this, previously used methods are either incomplete, i.e. not considering both the variation of the standard curve and that of the unknown samples (29, 30 ), approximative, and/or have been applied to very complicated law of mass action models for curve fitting $(23,28,31)$ seemingly requiring too much data-capacity for routine use. To date we have found no publication dealing with the complicated matter of calculating confidence intervals in the spline situation. The use of spline functions also increases the number of standard levels needed to obtain a sufficient number of degrees of freedom of the variance estimates. Anyhow, a single polynomial to approximate the standard curve simplifies the calculation of confidence intervals. In our application these calculations can be performed on a programmable desk top electronic calculator.

In our opinion, there are practical advantages in providing confidence intervals in addition to point estimates.

Especially in research work our evaluation method may improve the control of radioimmunoassay methods. Furthermore, in routine clinical practice, the physician can be offered an assay result with an indication of its reliability.

\section{Acknowledgements}

This project was supported by the Swedish Medical Research Council, project No B78-14X-02 829-09.

\section{References}

1. Challand, G. S. (guest editor) 1978, Automated calculation of radioimmunoassay results. Report of a meeting held at the Clinical Research Centre, Northwick Park Hospital, Harrow, Middlesex, on 13 December 1977, Ann. Clin. Biochem. 15, 123-135.

2. Rodbard, D., Bridson, W. \& Rayford, P. L. (1969), J. Lab. Clin. Med. 74, 770-781.

3. Rodbard, D. \& Hutt, D. M. (1974), in Radioimmunoassay and Related Procedures in Medicine Vol. I, 165-192, International Atomic Energy Agency, Vienna.

4. Wilkins, T. A., Chadney, D. C., Bryant, J., Palmström, S. H. \& Winder, R. L. (1978), in Radioimmunoassay and Related Procedures in Medicine Vol. I, 399-423, International Atomic Energy Agency, Vienna.

5. Täljedal, I.-B. \& Wold, S. (1970), Biochem. J. 119, 139-143.

6. Marschner, I., Erhardt, F. \& Scriba, P. C. (1974), in Radioimmunoassay and Related Procedures in Medicine Vol. I, 111-122, International Atomic Energy Agency, Vienna.
7. Lötz, A., Vogt, W., Popp, B. \& Knedel, M. (1976), Comput. Biomed. Res. 9, 21-30.

8. Vogt, W., Popp, B. \& Knedel, M. (1973), Z. Klin. Chem. 11, 438-445.

9. Rodbard, D. \& Catt, K. J. (1972), J. Steroid Biochem. 3, 255-273.

10. Malvano, R. \& Rolleri, E. (1975), Pathol. Biol. 23, 863-868.

11. Rodbard, D. (1974), Clin. Chem. 20, 1255-1270.

12. Ekins, R. P. (1974), Br. Med. Bulletin 30, 3-11.

13. Challand, G. S., Spencer, C. A. \& Ratcliffe, J. G. (1976), Ann. Clin. Biochem. 13, 354-360.

14. Rawlins, T. G. R. \& Yrjönen, T. (1978), Int. Lab., - 55-65.

15. Wold, S. (1974), Technometrics 16, 1-i1.

16. Nclte, H., vón zur Mühlen, A. \& Hesch, R. D. (19.76), J. Clin. Chem. Clin. Biochem. 14, 253-259.

17. Walker, W. H. C. (1977), Clin. Chem. 23, 384-402. 

18. Bergdahl, B., Dahlström, G., Molin, L. \& Bertler, A. (1979),
Acta Pharmacol. Toxicol. 45, 66-72. 19. Fieller, E. C. (1940), J. R. Stat. Soc. CIII, Suppl. VII, No. 1,
1-64.

20. Kubasik, N. P., Hall, J. L., Barold, S. S., Volosin, M. T. \& Sine, H. E. (1976), Chest 70, 217-220.

21. Rodbard, D. \& Frazier, G. R. (1975), in Methods in Enzymology vol. 37B, 3-22, Academic Press, New York.

22. Riggs, D. S. (1972), in The Mathematical Approach to Physiological Problems, 50-51, The Williams \& Wilkins
Comp., London.

23. Pilo, A. \& Zucchelli, G. C. (1975), Clin. Chim. Acta 64,

24. Bergdahl, B., Molin, L., Lindwall, L., Dahlström, G., Scherling, I.-L. \& Bertler, A. (1979), Clin. Chem. 25, 305-308.

25. Armitage, P. (1973), in The normal (or Gaussian) distribution, 76, Blackwell Scientific Publications, Oxford.
26. Scheffé, H. (1967), in The Analysis of Variance, John Wilcy \& Sons Inc. London.

27. Ekins, R. P. (1974), in Radioimmunoassay and Related Procedures in Medicine Vol. I, 91-109, International Atomic Energy Agency, Vienna.

28. Finney, D. J. (1976), Biometrics 32, 721-740.

29. Rodbard, D., Rayford, P. L., Cooper, J. A. \& Ross, G. T (1968), J. Clin. Endocrinol. 28, 1412-1418.

30. Rodbard, D. \& Cooper, J. A. (1970), in In Vitro Procedures with Radioisotopes in Medicine, 659-674, International Atomic Energy Agency, Vienna.

31. McHugh, R. B. \& Meinert, C. L. (1970), in Statistics in Endocrinology (McArthur, J. W. \& Colton, T., eds.), 399410, MIT Press, Cambridge.
L. Molin, Pharm. D.

Dept. of Clinical Pharmacology

University Hospital

S-581 85 Linköping Sweden 


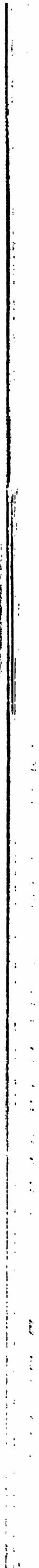

